\title{
Revictimización y procesamiento de la información: problemas en el estudio de las dificultades de razonamiento
}

\author{
REVICTIMIZATION AND INFORMATION PROCESSING: PROBLEMS IN THE STUDY OF REASONING \\ DIFFICULTIES
}

Dr. Miguel López Astorga (milopez@utalca.cl), Instituto de Estudios Humanísticos "Juan Ignacio Molina", Universidad de Talca (Talca, Chile)

\begin{abstract}
DePrince relates problems such as dissociation, revictimization and difficulties in social cognition. In particular, she states that individuals with dissociation or have been, by their own testimony, revictimized show obvious difficulties to solve selection tasks designed as social contracts or precautory problems. In my view, these facts mean that, if we accept the theories that there are mechanisms in human mind to regulate social exchanges and situations of risk, individuals with dissociation or revitimized may have altered such mechanisms. However, these theories have been critized and we have no evidence that they are valid. Therefore, in this paper, not admitting the existence of these mechanisms, I try to show that DePrince's outcomes can be interpreted from other perspectives.
\end{abstract}

Keywords: caution, dissociation, reasoning, revictimization, social contract

\section{Resumen}

DePrince establece relaciones entre problemas como la disociación, la revictimización y ciertas dificultades en la cognición social. En concreto, afirma que las personas que padecen de disociación o que han sufrido, según su propio testimonio, episodios de revictimización manifiestan evidentes dificultades para resolver tareas de selección planteadas como contratos sociales o como problemas de precaución. En nuestra opinión, esto significa que, si aceptamos las teorías que defienden que existen mecanismos en la mente humana que regulan los intercambios sociales y las situaciones de riesgo, las personas que han sido diagnosticadas con disociación o que han sido revictimizadas pueden presentar alteraciones de tales mecanismos. No obstante, esas teorías han sido cuestionadas y no contamos con pruebas concluyentes de que sean válidas. Por ello, en este trabajo, no admitiendo la existencia de los mencionados mecanismos, intentamos mostrar que los resultados de DePrince pueden ser interpretados desde otras perspectivas.

Palabras clave: contrato social, disociación, precaución, razonamiento, revictimización

\section{Introducción}

DePrince (2005) nos presenta una investigación en la que trata de hallar relaciones entre la teoría del trauma con traición, la disociación y la revictimización. Según nos cuenta DePrince, existe una teoría, 
denominada teoría del trauma con traición, que se remonta a los trabajos de Freyd $(1994,1996)$ y que se basa en la idea de que detectar comportamientos que violan relaciones básicas de intercambio social puede resultar poco productivo en determinadas circunstancias, como, por ejemplo, en ciertos casos de abuso infantil. Se argumenta que, si una víctima es consciente de un abuso perpetrado por alguien que, de manera habitual, es responsable de su cuidado y responde a tal abuso, el resultado lógico es que la víctima se aleje de la persona que comete el abuso y que desaparezca la relación de cuidado que ambos mantienen. El problema parece ser que, cuando la víctima depende del cuidador, la respuesta puede no ser percibida como adecuada, ya que el cuidador puede reaccionar proporcionando menos cuidado o incrementando aún más su agresividad. Por tanto, la supervivencia puede requerir que la víctima permanezca inconsciente de la traición, y ello sólo para garantizar que el cuidador abusador siga cumpliendo sus funciones de cuidado (1).

Es en este sentido en el que Freyd (1996), según interpreta DePrince, entiende que la disociación es un mecanismo para eliminar de la conciencia la información relacionada con los abusos, ya que, en su opinión, la exposición a situaciones traumáticas con un alto nivel de traición debe estar asociada a grados altos de disociación. Los traumas interpersonales pueden variar, desde su óptica, en función del grado de dependencia que exista entre la víctima y el victimario y, por consiguiente, en función del grado de traición que se produzca. $Y$ es que no es lo mismo que el abuso sea realizado por un individuo del que la víctima depende, situación en la que el abuso es asumido como una traición de nivel muy elevado, que por un extraño, situación en la que el abuso es vinculado con un nivel de traición marcadamente más bajo. A partir de estos supuestos, la teoría del trauma con traición defiende que las personas que han sufrido abusos con altos niveles de traición pueden presentar dificultades para detectar o ser conscientes de los abusos y que, por esta razón, existe un grado de probabilidad notablemente elevado de que sean revictimizadas.

El planteamiento parece ser, así, según lo expuesto, que un trauma percibido como una fuerte traición conduce a la disociación y que ésta incrementa las posibilidades de futuras revictimizaciones. De esta manera, puesto que la disociación, como es sabido, se encuentra vinculada con dificultades en el procesamiento de la información, DePrince alude a investigaciones como la de Cloitre, Scarvalone y Difede (1997) para recordarnos que se ha dicho que los individuos muy disociativos pueden ser menos conscientes de los riesgos del medio circundante, lo que, a su vez, puede incrementar también su riesgo de revictimización. De hecho, se han sostenido tesis como que los depredadores sexuales pueden reconocer fácilmente la vulnerabilidad de mujeres que manifiestan síntomas de disociación, por ejemplo, mirada confundida o distraída, y, de esta forma, captarlas como víctimas potenciales.

No obstante, en este contexto teórico, la hipótesis de DePrince parece ser que la disociación se encuentra relacionada de modo directo con procedimientos muy básicos con los que la información es procesada, y no necesariamente con situaciones sociales que requieren un procesamiento más complejo. Su propuesta es que, efectivamente, la disociación incrementa el riesgo de revictimización, pero defiende que lo hace, exclusivamente, porque se encuentra relacionada con cómo se procesa información social bastante elemental. En su opinión, las investigaciones previas al respecto, como, por ejemplo, la de Sandberg, Lynn y Matorin (2001), simplemente, han aplicado los paradigmas en los que a las mujeres se les solicita emitir juicios acerca de situaciones amenazantes que observan en viñetas que representan violaciones potenciales. Empero, desde su punto de vista, las dificultades de la disociación hacen referencia, como decimos, a procesos de asimilación de información básica, los cuales son mucho menos complicados que los implicados en evaluar amenazas en situaciones sociales representadas por viñetas. 
Para demostrarlo, recurre a versiones de la tarea de selección de las cuatro tarjetas de Peter Wason (1966, 1968). Utiliza versiones de tres tipos: abstractas, con contrato social y de precaución. Generalmente, las personas que realizan estos ejercicios suelen obtener muy malos resultados en las versiones abstractas y bastante mejores en las versiones con contrato social o de precaución. El propósito de DePrince parece, de esta forma, comprobar cómo reaccionan ante estas tareas individuos revictimizados y con disociación y hasta qué punto se verifican, en función de los resultados observados, las predicciones de la teoría del trauma con traición. Ella piensa que, si las personas con disociación o revictimizadas no responden igual que la población general ante estos ejercicios de razonamiento (ejecutando pobremente las versiones abstractas y de mejor modo las otras dos), sino que presentan problemas para resolver adecuadamente los tres tipos de versiones, su tesis relativa a que la disociación afecta a procesamientos básicos de información se verá confirmada.

Sin embargo, nosotros creemos que los resultados de un experimento de esta índole pueden conducir a conclusiones diferentes a las previstas por DePrince y que no pueden ser utilizados para probar tesis como la que pretende demostrar, pues existen enfoques teóricos que han analizado en profundidad la tarea de selección en general -y sus versiones con contratos sociales y de precaución en particular- y que parecen remitir a direcciones distintas a la que apunta DePrince. Pensamos que tales enfoques deben ser tenidos en cuenta, ya que la revisión de sus explicaciones de los aciertos y errores de los participantes en estos ejercicios puede guiarnos a maneras alternativas de entender la problemática de las personas con disociación o revictimizadas. $Y$ es que interpretar los resultados de DePrince desde los marcos de los enfoques teóricos mencionados puede concedernos la oportunidad de entender que los supuestos sobre la cognición humana de los que partimos nos abocan a interpretaciones diferentes de los resultados de los experimentos y, por tanto, a conclusiones distintas sobre la actividad mental de los participantes, sean éstos personas con disociación, revictimizadas o pertenecientes a la población general.

Es necesario, no obstante, no olvidar que algunas teorías han apelado a ciertos mecanismos mentales como los responsables de las respuestas mayoritarias de los participantes en la tarea de selección. Por tanto, si las personas que padecen disociación o que han sido revictimizadas no realizan las diferentes versiones de la tarea de selección del mismo modo que la población general (sino de manera sensiblemente peor), parece legítimo conjeturar, desde los supuestos de dichas teorías, que manifiestan algún tipo de déficit en tales mecanismos. Esta tesis, como vamos a comprobar, estaría en sintonía con los argumentos de DePrince y podría prestarle apoyo teórico. Sin embargo, la complejidad de este asunto es enorme, ya que, para aceptar esta idea, tenemos que asegurarnos de que lo que conocemos hasta el momento presente sobre la disociación y la revictimización nos autoriza a asumir que se vinculan con dificultades en mecanismos como los aludidos y, además, es preciso no obviar las críticas que, desde la literatura, se han presentado contra las teorías que han defendido la existencia de dichos mecanismos para explicar los resultados en la tarea de selección. Estos temas son los que vamos a enfrentar en lo que sigue, pero, antes de entrar directamente en la discusión, parece oportuno recordar en qué consisten exactamente las versiones abstractas, con contratos sociales y de precaución de la tarea de selección de las cuatro tarjetas de Peter Wason.

\section{Las tareas de selección abstractas, con contratos sociales y de precaución}

Como hemos indicado, DePrince nos habla de tres tipos de versiones de la tarea de selección, que son los que utiliza en su estudio, pero, por lo que a este asunto se refiere, podemos plantear dos observaciones: por una parte, existe, al menos, un tipo más, pudiéndose distinguir versiones abstractas, descriptivas, con 
contratos sociales y de precaución, y, por otra, en DePrince se confunden las versiones descriptivas con las abstractas.

Las versiones que DePrince denomina abstractas son, en realidad, las descriptivas. En estas versiones, se les presenta a los participantes una regla de razonamiento condicional (si-entonces) de este tipo: "If a train goes to Rochester, then it must be on Track 7" (DePrince 2005:128).

El participante ve cuatro tarjetas que representan cuatro trenes. Cada tarjeta indica, por un lado, dónde se dirige el tren y, por otro, en qué andén se encuentra. De este modo, en la primera tarjeta puede aparecer escrito Rochester, en la segunda puede figurar el nombre de otra ciudad, la tercera puede hacer alusión al andén 7 y la cuarta indicar otro andén distinto. Ante este escenario, lo que se le solicita al participante que haga es seleccionar las tarjetas necesarias para comprobar si la regla condicional es correcta o no. La respuesta apropiada es elegir, en este ejemplo, la tarjeta en la que aparece Rochester (puede hacer referencia por detrás a un andén distinto al 7) y la tarjeta en la que figura un andén distinto al 7 (puede señalar en su otra cara que ese tren va a Rochester). La tarjeta con una ciudad diferente a Rochester no interesa (la regla no establece nada con respecto a otras ciudades y, por tanto, los trenes que van a ellas pueden situarse en cualquier andén, sea o no el 7), como tampoco aquella en la que figura una alusión al andén 7 (la regla no obliga a que en él sólo pueda haber trenes que van a Rochester, sino, únicamente, a que los trenes con destino a Rochester se sitúen en él, lo que significa que no quebranta la regla el hecho de que en el andén 7 esté un tren que no vaya a Rochester).

Como decimos, no se puede afirmar, en rigor, que ésta es una versión abstracta de la tarea de selección de las cuatro tarjetas de Peter Wason. Ese término se reserva, habitualmente, en la literatura, para versiones semejantes a las originales con las que trabajó el propio Wason. En estas últimas versiones, la regla suele vincular a una vocal con un número par y en las tarjetas se observa una letra en dos de ellas y un número en las otras dos. En las tarjetas con letras aparece una vocal en una de ellas y una consonante en la otra, mientras que en las tarjetas con números figura un número par en una de ellas y un número impar en la otra. El ejemplo que nos presenta DePrince tiene, como se puede observar, un contenido temático y, por ello, no es correcto referirse a él como un ejemplo de una versión abstracta. Es más apropiado indicar que se trata de una versión descriptiva.

No obstante, exclusivamente con el propósito de no generar confusiones, vamos a referirnos a este tipo de versión utilizando el término atribuido a él por DePrince y, por tanto, cuando mencionemos en las páginas siguientes a las versiones abstractas, estaremos aludiendo verdaderamente a versiones descriptivas como la del tren y la ciudad de Rochester que acabamos de comentar.

En cualquier caso, la problemática de estas versiones es que, en la mayor parte de los casos, los participantes no las resuelven adecuadamente, pues muy pocas veces eligen las tarjetas correctas. La situación cambia cuando se trata de versiones con contratos sociales o de precaución, ya que en ellas se observan porcentajes significativamente más elevados de elección correcta de tarjetas. Las versiones con contratos sociales son aquellas en las que, si bien se mantiene exactamente la misma estructura lógica, la regla refleja una situación de contrato social, es decir, una situación en la que un individuo puede conseguir un beneficio si cumple con un requerimiento. Un ejemplo de regla en este tipo de versión puede ser: "If you get someone else to cover a work shift for you, then make a donation to the community fund" (DePrince 2005:128-129). 
Naturalmente, en este escenario, las tarjetas reflejan si se encontró o no a alguien para cubrir el turno de trabajo y si se hizo o no una donación al fondo de la comunidad. Los resultados en versiones de esta índole, reiteramos, mejoran notablemente.

Exactamente lo mismo sucede generalmente en las versiones con precaución, pues también en ellas los participantes tienden a inclinarse por las tarjetas válidas. Estas versiones conservan igualmente la estructura de las versiones abstractas, pero presentan un enunciado condicional como una regla diseñada para proteger la seguridad de las personas. Una regla de precaución característica puede ser ésta: "If you surf in cold water, then wear a wetsuit" (DePrince 2005:129).

Obviamente, en una versión como ésta las tarjetas tienen que hacer referencia a si se está navegando o no por agua fría y a si se está utilizando o no un traje de neopreno.

Ante estos hechos, han surgido teorías que explican las diferencias de resultados entre los tipos de versiones indicados recurriendo a la hipótesis de que existen mecanismos mentales relacionados con dominios específicos que, al margen de las capacidades lógicas de los individuos, permiten ejecutar correctamente ciertas versiones de la tarea de selección. A esta dirección apunta la teoría de los contratos sociales, defendida en trabajos como Fiddick, Cosmides y Tooby (2000) y que señala que los seres humanos disponemos de mecanismos mentales producto de nuestra evolución que nos permiten enfrentarnos adecuadamente a situaciones sociales de intercambio y detectar, sin mayores dificultades, a las personas que no actúan apropiadamente en tales relaciones, es decir, a las personas que violan las normas o los acuerdos disfrutando de beneficios sin cumplir ningún requerimiento. Esto se traduce, en el caso del ejemplo de versión de la tarea de selección con contrato social indicado más arriba, en que los mecanismos mentales con los que contamos los seres humanos para identificar tramposos nos permiten notar rápidamente que un individuo que quebranta la norma es el que consigue a alguien para que le cubra un turno de trabajo y, sin embargo, no realiza donación alguna al fondo de la comunidad. Es por esta razón por la que, en tareas de selección como ésta, los participantes se inclinan mayoritariamente por las tarjetas correctas, esto es, como acabamos de mencionar, por la tarjeta en la que figura que se encontró a otra persona para que hiciera un turno de trabajo y por la tarjeta que refleja que no se entregó nada al fondo comunitario.

Pero en trabajos como Fiddick et al. (2000) se avanza un poco más y se defiende que no sólo existen mecanismos mentales para regular los contratos sociales, sino también para reaccionar adecuadamente ante situaciones que implican riesgo o peligro. De esta manera, Fiddick et al. (2000) parecen argumentar que, para el progreso y la supervivencia, el proceso evolutivo no tuvo que dotar a los seres humanos únicamente con sistemas que le permitieran de manera sencilla detectar a los sujetos que rompen los acuerdos y los tratos, sino que también tuvo que otorgarles algoritmos para que no les resultara complejo notar en qué situaciones existe riesgo. Así, desde esta óptica, puede entenderse también, en su opinión, por qué los participantes en versiones de la tarea de selección con precaución eligen, habitualmente, las tarjetas adecuadas, es decir, por qué en versiones como la del traje de neopreno comentada en líneas anteriores los participantes notan rápidamente y sin demasiados esfuerzos que las tarjetas válidas son las relativas a navegar en agua fría y a no usar un traje de neopreno.

Si tenemos en cuenta estos planteamientos que sostienen la existencia de mecanismos especializados en dominios específicos en nuestra mente, la investigación de DePrince puede ser analizada desde un ángulo 
que, sin hacer referencia directa a él, parece, como se va a poder apreciar más abajo, adoptar al exponer sus conclusiones. La idea vendría a ser, en este sentido, que, si las personas con disociación o revictimizadas de su estudio no presentan los mismos resultados positivos que la población general en las versiones con contratos sociales y de precaución (sino que en ellas los porcentajes de selección correcta para estos participantes no difieren mucho de los que se suelen obtener en las versiones abstractas), puede argumentarse que la disociación y la revictimización pueden estar vinculadas con ciertas limitaciones o alteraciones en los mecanismos mentales evolutivos específicos para detectar infractores y para identificar riesgos o peligros.

No obstante, como ya apuntamos, la complejidad de estas temáticas es grande, puesto que, como vamos a comprobar más adelante, en el momento presente, no contamos con un consenso teórico con respecto a los mecanismos mentales mencionados. Diversos autores los cuestionan y no está clara su verdadera existencia. Esto puede significar, sin duda, que, al menos, hasta el momento, no disponemos de bases o fundamentos lo suficientemente firmes como para interpretar de manera indiscutible los resultados de experimentos como el de DePrince. Empero, con carácter previo a la revisión de la problemática que envuelve a la detección de infractores y a la identificación de riesgos en los seres humanos, puede ser apropiado profundizar en determinados detalles de la investigación de DePrince.

\section{Los hallazgos de DePrince}

Sobre la base de la teoría del trauma con traición, DePrince se propone comprobar estas predicciones:

- La presencia de un alto índice de trauma con traición en edades anteriores a los dieciocho años se encuentra asociada con la disociación patológica.

- La presencia de tanto un alto índice de trauma con traición en edades anteriores a los dieciocho años como de disociación patológica se encuentra asociada con la revictimización después de los dieciocho años.

- Los individuos revictimizados después de los dieciocho años cometen más errores en versiones de la tarea de selección con contratos sociales y de precaución que los sujetos no revictimizados.

Con el propósito de verificar si sus predicciones son ciertas, DePrince trabaja con estudiantes de la Universidad de Denver con edades comprendidas entre los dieciocho y los treinta y tres años y utiliza versiones de la tarea de selección de las cuatro tarjetas de Peter Wason abstractas, con contratos sociales y de precaución procedentes de Stone, Cosmides, Tooby, Kroll y Knight (2002). Para medir el grado de disociación de los participantes, recurre a la Escala de Experiencias Disociativas (Bernstein y Putnam 1986). Por su parte, para medir el nivel de trauma con traición de los mismos, utiliza el Breve Estudio de Trauma con Traición, tomado de un documento no publicado de Goldberg y Freyd en el momento en el que DePrince plantea su investigación (2). Igualmente, emplea la Lista-40 de Síntomas de Trauma (Briere y Runtz 1989) con el propósito de controlar y evaluar experiencias asociadas a eventos traumáticos.

El Breve Estudio de Trauma con Traición sirvió, además, para constituir el grupo de participantes que había sufrido revictimización. Así, se notó que el grupo revictimizado formado de esta manera obtuvo una puntuación más elevada en la Lista-40 de Síntomas de Trauma. Otros datos relevantes obtenidos fueron, por ejemplo, que el trauma con traición antes de los dieciocho años se encontraba asociado con la disociación patológica y con la revictimización después de los dieciocho años y que la disociación patológica también lo estaba con la revictimización después de los dieciocho años. 
Sin embargo, lo que más nos interesa en estas páginas es que la revictimización pareció tener efectos significativos en la ejecución de problemas con contratos sociales y de precaución, pues el grupo de participantes que habían sido revictimizados cometió más errores que el grupo de participantes que no habían sido revictimizados en las versiones de la tarea de selección de las cuatro tarjetas con contratos sociales y de precaución. Esto no sucedió con las versiones abstractas, lo cual fue interpretado por DePrince como una evidencia de que el grupo de participantes revictimizados no manifestó limitación alguna con respecto al razonamiento general, sino únicamente con respecto a reglas condicionales con contenido social o relativo a normas de seguridad. $Y$ es que DePrince se inclinó a pensar que, puesto que las diferencias observadas entre los dos grupos se basaron en problemas que, si bien estaban planteados en términos de contratos sociales o de reglas de precaución, no hacían referencia a un contenido relacionado con situaciones de abuso, la revictimización puede tener vínculos con la alteración de las capacidades globales para procesar información social o vinculada con la seguridad.

Otro elemento que pareció tener relación con una mala ejecución de versiones con contratos sociales y de precaución fue la disociación patológica, no mostrándose significativos al respecto ni la influencia del trauma con traición antes de los dieciocho años ni los problemas generales medidos por la Lista-40 de Síntomas de Trauma. De este modo, reconociendo algunas limitaciones en su estudio y planteando algunas direcciones a las que debería encaminarse la investigación futura en estos ámbitos, DePrince sugiere, según entendemos, que lo que su trabajo revela de manera incuestionable es que la disociación patológica es un elemento que puede alterar la cognición social y, por tanto, incrementar en los individuos el riesgo de ser revictimizados.

\section{Disociación patológica, revictimización y mecanismos mentales de dominio específico}

Si analizamos estos hallazgos de DePrince en función del marco de las teorías mencionadas más arriba que postulan la existencia de algoritmos o sistemas mentales especializados en dominios específicos, como pueden serlo los relativos a los ámbitos que estamos estudiando en este trabajo, esto es, el de los contratos sociales y el de las situaciones de riesgo, las conclusiones son obvias y no se hallan, en nuestra opinión, muy alejadas de las que expone ella misma, no siendo, por tanto, complejo conjeturar cómo los defensores de tales teorías podrían interpretar sus resultados. Para ellos, podría suponerse, las experiencias traumáticas con traición antes de los dieciocho años de edad podrían provocar disociaciones patológicas (3) que afectarían a los mecanismos mentales que se activan cuando se está ante un intercambio social o una circunstancia que requiere precaución para salvaguardar la seguridad. La alteración de dichos mecanismos, por su parte, significaría que las personas que han sufrido traumas con traición se caracterizarían por una mayor vulnerabilidad y por presentar menores posibilidades de enfrentarse a los retos del entorno con éxito, lo que provocaría que aumentaran significativamente sus probabilidades de ser revictimizadas.

No obstante, las dificultades de una interpretación de la investigación realizada por DePrince en esta dirección consisten, como hemos apuntado, en que las teorías que defienden la existencia de mecanismos mentales de dominio específico han sido criticadas en diversas ocasiones, pudiéndose encontrar una gran variedad de argumentos contra ellas en la literatura. La mayor parte de las críticas inciden en que no es legítimo comparar los resultados de participantes en tareas de selección abstractas con los que se obtienen 
en tareas de selección con contratos sociales o de precaución, ya que entre estos tipos de tareas existen importantes diferencias.

Vamos a hacer referencia a continuación a las críticas que más se pueden relacionar, en nuestra opinión, con la problemática que estamos abordando en estas páginas. No obstante, a título ilustrativo, podemos comenzar mencionando también un cuestionamiento muy difícil de rebatir y que parece derribar de manera implacable los cimientos fundamentales de las teorías defensoras de mecanismos mentales evolutivos de dominio específico. Se trata de un argumento presente en Beller y Spada (2003) y que señala que, incluso si en algún momento lográramos demostrar de modo concluyente que existen mecanismos mentales de dominio específico, nunca podríamos estar totalmente seguros de que, como producto de la evolución, nacemos con ellos, puesto que también cabría siempre la posibilidad de haberlos adquirido por causas ambientales, por ejemplo, a partir del proceso educativo. Esta idea ya, por sí misma, permite pensar en otra interpretación diferente a la que nos ofrece DePrince de sus resultados, esto es, en que las dificultades cognitivas relacionadas con la revictimización son más generales que las que ella plantea, ya que el hecho de que la acción de los mecanismos mentales específicos en las personas revictimizadas no sea la adecuada podría implicar problemas de aprendizaje en el transcurso de su educación que han impedido el desarrollo de determinadas facultades -en este caso, las necesarias para afrontar situaciones de contrato social o de riesgo- presentes en la población general.

Pero consideramos que no debemos quedarnos únicamente con esta idea, pues se han presentado diversos trabajos en los que no sólo se duda del carácter evolutivo de los mecanismos mentales específicos, sino también de su existencia. Tal puede ser, por referirnos a algunos trabajos significativos en el área, el caso de Sperber y Girotto (2002) y Girotto y Tentori (2008). Desde estos enfoques, se plantea que las versiones de la tarea de selección con contratos sociales no son, verdaderamente, el mismo tipo de ejercicio que las versiones abstractas, pues, según interpretamos, las versiones abstractas son, efectivamente, tareas de razonamiento condicional, pero las versiones con contratos sociales son tareas en las que, simplemente, hay que identificar las tarjetas que corresponden a una categoría indicada, lo cual es una labor mucho más sencilla. Esto significa que una tarea abstracta es, cognitivamente, bastante más difícil de resolver que una tarea con contrato social. En nuestra opinión, Sperber-Girotto (2002) y Girotto-Tentori (2008) están, de modo indiscutible, en lo cierto al afirmar que la complejidad de las tareas abstractas es claramente mayor, y ello podemos observarlo con evidencia si atendemos a las traducciones de ejemplos indicados más arriba. Pensemos primero en una regla como ésta:

A. Si un tren va a Rochester, entonces debe estar en el andén 7.

En realidad, no es nada simple entender el auténtico sentido de una tarea de selección abstracta que proponga una regla semejante a A. El participante puede, por poner el caso, interpretarla de manera bicondicional y no pensar sólo que los trenes que van a Rochester se encuentran en el andén 7, sino también que los trenes que se hallan en el andén 7 son exclusivamente los que se dirigen a Rochester. Esto es menos probable que ocurra ante enunciados similares al siguiente:

B. Si consigues a alguien para cubrirte un turno de trabajo, entonces haz una donación al fondo de la comunidad. 
Sería muy extraño que el participante en una tarea de selección con contrato social con una regla como B entendiera que esta regla no sólo establece que, cuando se consigue a alguien para cubrir un turno de trabajo, hay que hacer forzosamente una donación a la comunidad, sino también que, cuando se hace una donación a la comunidad, hay obligatoriamente que conseguir a alguien para que cubra un turno de trabajo. Por consiguiente, aquí es realmente complejo que se produzca una interpretación bicondicional de la regla, pues su sentido es mucho más obvio.

Sin embargo, la idea de Sperber-Girotto (2002) y de Girotto-Tentori (2008) apunta a que un participante, ante la solicitud de comprobar la verdad o la falsedad de una regla del tipo de $\mathrm{B}$, sólo tiene que pensar en la categoría de individuos que pueden infringirla, esto es, en aquellos sujetos que han conseguido a alguien para cubrir un turno de trabajo y que, empero, no han hecho una donación al fondo de la comunidad, lo cual lo dirige de manera casi directa a las tarjetas adecuadas (la que indica que se encontró a alguien para cubrir un turno y la que señala que no se hizo ninguna donación). A nuestro juicio, este planteamiento de SperberGirotto y de Girotto-Tentori debe ser completado con una explicación de por qué las tareas abstractas no pueden tornarse para el participante de manera tan simple en tareas de categorización. Tal explicación pueden proporcionárnosla circunstancias, propias de reglas abstractas como $\mathrm{A}$, similares a la que acabamos de describir, esto es, similares al hecho de que es más complejo entender su significado exacto, ya que tales reglas pueden, por ejemplo, no ser interpretadas como condicionales, sino como bicondicionales.

Estas consideraciones nos conducen a otras críticas contra las teorías defensoras de mecanismos evolutivos de dominio específico en la mente humana y, en concreto, a las propuestas en trabajos como López (2010, 2011). En estos trabajos se plantea, básicamente, que la mayor parte de los experimentos que han presentado los autores que sostienen la existencia de mecanismos mentales de dominio específico para intentar demostrar sus supuestos adolecen de problemas importantes en sus diseños, ya que las tareas con contratos sociales y de precaución que emplean para comparar sus resultados con los de versiones abstractas son tareas que poseen ciertas características que facilitan su comprensión con respecto a las abstractas (por ejemplo, un contexto más enriquecido y obvio o unas instrucciones que dirigen de un modo más sencillo a la respuesta correcta). Así, sus argumentos nos revelan que, aunque existieran realmente mecanismos mentales para regular los contratos sociales y para enfrentarse a situaciones que implican peligro, ello aún no está demostrado y que, por tanto, continúa estando justificado pensar que el funcionamiento de las capacidades generales de razonamiento es el que explica las diferencias en la ejecución de las distintas versiones de la tarea de selección de las cuatro tarjetas.

Críticas como éstas nos encaminan, a su vez, a plantearnos, como otra posibilidad, que el ámbito que realmente se encuentra alterado en las personas que han sido revictimizadas no se encuentra relacionado con mecanismos mentales especializados de ningún tipo y, yendo más allá que DePrince, que las disociaciones patológicas que aumentan el riesgo de revictimización no sólo inciden en capacidades para procesar información social o relativa a situaciones de riesgo, sino también en capacidades aún más generales para interpretar información, sea ésta de la índole que sea. Y es que las críticas contra las teorías defensoras de mecanismos evolutivos de dominio específico en la mente humana a las que hemos hecho referencia sugieren que las diferencias en los resultados de los participantes cuando se enfrentan a tareas de selección abstractas, con contratos sociales o de precaución no se deben necesariamente a la naturaleza del contenido semántico de los enunciados, sino a aspectos más amplios y abarcadores vinculados con la comprensión y la interpretación de instrucciones y mensajes. Evidentemente, profundizando en estos aspectos más amplios podemos arribar a concepciones diferentes de la revictimización. 
Como hemos señalado, los enfoques críticos que acabamos de exponer no son los únicos que podríamos haber mencionado, pero sí son, desde nuestra óptica, los que, sin sobrepasar los propósitos de este trabajo revisando una cantidad excesivamente elevada de investigaciones, nos posibilitan extraer, en mayor medida, conclusiones a partir de los resultados de DePrince. En este sentido, podemos decir que, si tuviéramos en cuenta, por ejemplo, las ideas fundamentales sobre las que gravitan trabajos como López $(2010,2011)$, ello nos permitiría estar en condiciones de afirmar que en las versiones de la tarea de selección con contratos sociales o de precaución se obtienen porcentajes más elevados de selección correcta porque las reglas que suelen incluirse en ellas son de más fácil interpretación y muestran de modo más nítido al participante que establecen una relación condicional entre un antecedente y un consecuente. Las personas que han sido revictimizadas, sin embargo, no serían, según estos supuestos, sensibles, de la misma manera que el resto de los individuos, a las características que facilitan la comprensión de estas tareas y, por esta razón, desde esta perspectiva, resultaría necesario adentrarse un poco más en el análisis de las facultades cognitivas que realmente se ven implicadas en la resolución de una versión de la tarea de selección. A esta labor especulativa dedicamos el apartado siguiente, con el propósito de mostrar con mayor claridad que los datos que arrojan ejercicios de razonamiento como la tarea de selección no nos permiten inferir, por sí mismos, conclusiones unívocas.

\section{Procesamiento de la información y revictimización}

Un estudio esencial en la discusión que rodea a la tarea de selección de las cuatro tarjetas de Peter Wason, y que es el que presta apoyo teórico a los planteamientos básicos presentes en López $(2010,2011)$, es el de Almor y Sloman (2000). Almor y Sloman presentaron en su trabajo un importante hallazgo. Con sus experimentos, descubrieron que en los participantes en las distintas versiones de la tarea de selección se podían distinguir dos fases cognitivas distintas. Una primera fase era la de procesamiento, en la que el participante interpretaba la información que se le transmitía y elaboraba representaciones mentales a partir de tal interpretación, y la segunda era la que verdaderamente podía denominarse de razonamiento.

Almor y Sloman llegaron a estas conclusiones porque plantearon a sus participantes versiones de la tarea de selección en las que la regla no se adecuaba correctamente a las instrucciones. Narraban una historia a los participantes y luego los enfrentaban a cuatro tarjetas y a una regla relacionada con la historia. La regla, en algunas condiciones experimentales, tenía el antecedente y el consecuente invertidos, de manera que no resultaba del todo compatible con el escenario propuesto. No obstante, tras finalizar su labor, Almor y Sloman preguntaron a sus participantes por la regla que aparecía en la tarea y la respuesta mayoritaria que recibieron fue, sorprendentemente, no la regla tal y como ellos la habían presentado, sino con el antecedente y el consecuente rotados, lo cual la compatibilizaba con la historia narrada. Del mismo modo, notaron que las tarjetas que eligieron sus participantes eran las correctas para la regla que recordaban, no para la original.

Estos hechos provocaron que sostuvieran, como hemos indicado, que se pueden diferenciar dos fases distintas en la actividad intelectual de una persona que se enfrenta a la tarea de selección, un fase en la que se procesa y otra en la que se razona. Partiendo de estas conclusiones, en López $(2010,2011)$ se defiende que la tarea de selección no es el ejercicio más idóneo para estudiar el razonamiento humano, ya que las causas por las que un participante realiza una elección errada de tarjetas pueden estar más relacionadas con la fase de procesamiento que con la de razonamiento, o, dicho con términos más sencillos, pueden deberse a que no han entendido la tarea, y no a una supuesta incapacidad para realizarla. 
Si asumimos estos argumentos, es válido afirmar que la disociación patológica que conduce a la revictimización puede afectar y condicionar a la primera fase propuesta por Almor y Sloman y, por consiguiente, dificultar en el individuo el procesamiento oportuno y la interpretación apropiada de las instrucciones. Esto, a nuestro juicio, es, en buena medida, consistente con las conclusiones de DePrince, ya que ella parece indicar que las dificultades asociadas a la disociación y a la revictimización se encuentran relacionadas con problemas en el procesamiento de la información (no olvidemos tampoco, en este sentido, que la disociación, tal y como nos recuerda DePrince, se define como la falta de integración de aspectos generalmente conectados en el procesamiento de la información).

Pero DePrince restringe el ámbito afectado en los individuos revictimizados, pues, aunque acepta que provoca dificultades globales, limita éstas, como hemos señalado, a las dimensiones relacionadas exclusivamente con la información social o relativa a la seguridad. Sin embargo, nosotros creemos que esta restricción a estas áreas específicas no puede sostenerse solamente en virtud de sus resultados, los cuales, como se está apreciando, admiten otras conclusiones. Naturalmente, se puede intuir y especular que la disociación en personas revictimizadas incide sólo en el procesamiento de información referente a contratos sociales o a situaciones de riesgo, pero, en rigor, el comportamiento de los participantes de DePrince deja abierta la posibilidad de que, en realidad, altere aspectos más generales de la actividad cognitiva, como los vinculados con la información referida a cualquier ámbito.

DePrince rechaza esta posibilidad porque no existen diferencias significativas entre los resultados de los participantes revictimizados y los de los participantes que no han vivido tal experiencia en la ejecución de las versiones abstractas de la tarea de selección que ella propone. Así, parece deducir, a partir de esta igualdad, que no existe tampoco una distancia notable entre las personas que han sufrido situaciones de revictimización y las que no se han visto en tales situaciones en lo que al estado de sus capacidades generales se refiere, circunscribiendo las distinciones sólo a escenarios de intercambio social o de situaciones amenazantes. No obstante, aquí podemos estar ante un importante error metodológico de DePrince que puede, indirectamente, fortalecer cualquier otra explicación alternativa de sus resultados. Hay que tener en cuenta que los resultados en la ejecución de tareas abstractas de los dos grupos son similares porque no se seleccionan mayoritariamente las tarjetas correctas en ninguno de los dos, y es, para nosotros, un poco complejo aceptar la tesis de que las capacidades generales del grupo de personas revictimizadas no se encuentran alteradas porque ejecutan estos ejercicios de un modo tan negativo como la población general. Es habitual que una persona no revictimizada no resuelva adecuadamente una tarea de selección abstracta, pero que no lo haga tampoco una persona revictimizada no demuestra que sus capacidades generales estén en óptimo estado, pues, al fin y al cabo, no ejecuta bien el ejercicio. Y es que sabemos que lo previsible es que una persona con sus capacidades cognitivas generales operando sin dificultades no elija las tarjetas adecuadas en tareas de selección abstractas, pero no podemos concluir por ello, como parece hacer DePrince, que las personas que se equivocan en la misma medida que la población general en su elección de tarjetas no manifiestan tampoco ningún déficit en sus capacidades generales de razonamiento. Simplemente, no podemos saber nada al respecto a partir de este hecho. De este modo, no parece una buena opción atender a los resultados de las versiones abstractas de la tarea de selección para discriminar entre personas sin problemas en sus facultades intelectuales y personas con dichas facultades limitadas, ya que, en ambos casos, se va a ofrecer, en la mayor parte de las ocasiones, una respuesta errada, esto es, una elección incorrecta de tarjetas. Por tanto, no consideramos que el que no existan diferencias entre los 
resultados de ambos grupos, el de las personas revictimizadas y el de las no revictimizadas, en las tareas abstractas en la investigación de DePrince aporte información relevante para la problemática que estamos analizando, ni que suponga un apoyo para las tesis de DePrince en detrimento de otras posibles.

Pero, por otra parte, puesto que no está claro que existan mecanismos mentales de dominio específico, no estamos tampoco completamente autorizados a relacionar las dificultades de las personas revictimizadas con dominios particulares como el de los contratos sociales o el de las situaciones que requieren precaución (lo cual podría apoyar la interpretación de DePrince), independientemente de sus resultados en tareas de selección con contratos sociales o de precaución. Ciertamente, críticas como la de Beller y Spada (2003) pueden ser obviadas, ya que puede argumentarse que, aunque los mecanismos de dominio específico no sean un producto del proceso evolutivo del ser humano, disponemos de ellos a causa de nuestro aprendizaje y se encuentran limitados en las personas que han sufrido revictimización. Empero, no se puede soslayar el hecho de que algunos planteamientos contra la idea de la existencia de los mecanismos de dominio específico son bastante firmes. En función de estos planteamientos, podemos decir que, como hemos apuntado en el apartado anterior, hasta el momento, no contamos con evidencia concluyente que nos conduzca a aceptar la acción de mecanismos de dominio específico en la actividad cognitiva humana. Los simples resultados en distintas versiones de la tarea de selección no son un apoyo sólido para los defensores de la existencia de tales mecanismos, ya que las diferencias en la ejecución, también lo hemos indicado, pueden deberse a múltiples factores.

Así, parece legítimo plantear, a partir de los resultados de DePrince, por lo menos, una explicación alternativa en un sentido completamente diferente, pues nos permiten defender que las personas revictimizadas manifiestan ciertos déficits para realizar tareas de selección que la población general suele resolver adecuadamente porque están expresadas de tal manera que es posible comprender perfectamente su sentido y el significado de su regla. Si admitimos que esta comprensión se produce debido a que, por el contexto, el participante entiende que se trata de una regla condicional y así la considera (y no, por ejemplo, como bicondicional), el que las personas que han sufrido revictimización no logren ejecutarlas adecuadamente puede significar únicamente que presentan problemas para la comprensión de mensajes y discursos. Por ello, podría proponerse la hipótesis de que a las personas revictimizadas no sólo les resulta complejo realizar tareas de selección con contratos sociales o de precaución, sino también cualquier otro ejercicio de razonamiento que requiera una cierta concentración y un determinado nivel de esfuerzo intelectual para procesar sus instrucciones, pues es válido defender, en base a los resultados de DePrince, que las dificultades de estas personas se encuentran en la primera fase planteada por Almor y Sloman, la de elaboración de representaciones mentales. De este modo, podría defenderse que un escenario cuya información es, en general, perfectamente procesada por la población general puede resultar complejo de entender para las personas revictimizadas, quizás, por ser difícil para ellas, por ejemplo, prestar la atención debida o relacionar información diversa, independientemente de que ésta esté referida al ámbito social, al del riesgo y el peligro o a cualquier otro.

\section{Conclusiones}

En muchas ocasiones, y en las más variadas áreas, se vinculan de manera inmediata los resultados que se obtienen con lo que parece intuitivamente más obvio. Esto es lo que, a nuestro juicio, sucede en el trabajo de DePrince, en el que se llega a la conclusión de que lo que sus resultados demuestran es que las personas revictimizadas manifiestan problemas en lo relativo al razonamiento acerca de las relaciones sociales y de 
las situaciones de peligro. No obstante, esta conclusión puede ser apresurada, ya que, de alguna manera, conduce, aunque DePrince parece no tenerlo en cuenta (o, al menos, no lo explicita), a aceptar supuestos, como los relativos a dominios mentales específicos referentes, precisamente, a las relaciones sociales y a las situaciones de peligro, que han sido, o están siendo, cuestionados en la literatura por diversos autores.

Así, entendemos que DePrince no recurre, desde el punto de vista metodológico, a la perspectiva más adecuada, ya que se queda exclusivamente con la interpretación que se deriva superficial y directamente de los datos experimentales que obtiene y no se plantea la posibilidad de que la acción de factores más profundos incida en dichos datos. Desde su punto de vista, si las personas revictimizadas presentan dificultades para resolver tareas de selección en términos de contratos sociales o de situaciones de riesgo, ello debe obedecer, simplemente, a dificultades en capacidades estrechamente relacionadas con situaciones semejantes a las descritas en tales tareas, sin barajar perspectivas teóricas más amplias que incluyan a esas capacidades en ámbitos generales. En este sentido, puede pensarse que la dirección que toma la investigación de DePrince, además de no ser la más apropiada, puede dificultar la identificación o el descubrimiento de otros ámbitos implicados en la cognición que pueden verse limitados en el caso de los participantes que ella estudia.

Puede, claro está, que la explicación que nos ofrece DePrince sea la correcta, pero no podemos aceptarla sin una demostración del supuesto del que, consciente o inconscientemente, parte, esto es, del supuesto de que existen dominios específicos de razonamiento en la mente humana que funcionan de manera autónoma y sin relación con otros dominios. Mientras no contemos con tal demostración, proceder como lo hace DePrince puede ser obviar todo el cúmulo de posibilidades interpretativas que puede aportarnos una visión global de la actividad mental humana.

Y es que, generalmente, aunque, en el caso de la tarea de selección de las cuatro tarjetas, especialmente, los datos estadísticos por sí solos no nos revelan mucho. Son necesarias construcciones teóricas consistentes con ellos para tratar de entender lo que nos muestran. Así, es posible, como hemos mostrado en las páginas precedentes, una explicación distinta, y coherente con otros enfoques de la literatura, para los resultados de DePrince desde una perspectiva más integradora de las facultades cognoscitivas, pues se puede pensar que las capacidades que se ven condicionadas o limitadas por la disociación patológica asociada a la revictimización no son de índole específica y que se encuentran vinculadas con la fase previa de procesamiento de información de la que nos hablan Almor y Sloman. Por supuesto, adoptar una perspectiva como ésta requiere continuar profundizando en la investigación y descubrir las causas de estos hechos, con el objetivo de establecer si se deben a problemas de concentración, a una imposibilidad para integrar la nueva información en el bagaje de conocimientos del sujeto o a otros factores completamente distintos. En cualquier caso, lo que es absolutamente necesario, a nuestro juicio, es tener presente que, con los datos con los que hoy contamos, limitar el estudio a las dimensiones sociales y de respuesta a los riesgos, usando versiones de la tarea de selección con contratos sociales o de precaución, puede ser problemático y, en temáticas como la de la disociación patológica o la revictimización, conducir a sesgos en los análisis y en las argumentaciones.

Tenemos que aceptar que el trabajo de DePrince abre muchas posibilidades al establecer relaciones entre el trauma con traición, la disociación y la revictimización, pero debemos tener en cuenta, igualmente, que la tarea de selección es uno de los ejercicios más controvertidos a los que se ha enfrentado la ciencia cognitiva y el estudio del razonamiento humano en la época contemporánea. No existe en el presente, después de 
varias décadas de discusión y de luchas esgrimiendo resultados experimentales, prácticamente ningún consenso claro con respecto a ella (4). Cada autor interpreta el comportamiento de sus participantes en esta tarea en función del marco teórico que asume y el enfoque que defiende la existencia de mecanismos mentales de dominio específico sólo es uno más de los que se han presentado para intentar explicar el hecho de que, aunque conserven la misma estructura lógica, algunas de sus versiones no son ejecutadas de un modo correcto por sus participantes.

Estas circunstancias deberían haber sido tenidas en cuenta por DePrince, pues, aunque ella, insistimos, no analiza la problemática de los mecanismos mentales de dominio específico ni explícitamente asume los supuestos de los autores que sostienen su existencia (se limita a citar trabajos de tales autores para solamente afirmar que las versiones con contratos sociales o de precaución suelen obtener mejores resultados que las abstractas), parece que, de alguna forma, acepta sus tesis fundamentales, puesto que considera que sus resultados muestran déficits en la comprensión de información social o relativa a peligros, y no en la comprensión de información general.

Se tornan oportunas, por tanto, discusiones epistemológicas profundas acerca de los diseños experimentales referentes a estas temáticas para verificar lo que realmente demuestran sus resultados. Nuestro propósito ha sido, en este sentido, principalmente, mostrar a lo largo de estas páginas que la investigación de DePrince puede no probar necesariamente lo que ella cree y que las selecciones de sus participantes en sus versiones de la tarea de selección son interpretables en otros sentidos si atendemos a la literatura acerca de dicha tarea. Parece, de este modo, esencial seguir estudiando la problemática de la tarea de selección antes de utilizarla en experimentos como el de DePrince, ya que los resultados que se pueden obtener con ella admiten interpretaciones diversas dependiendo del enfoque teórico que adoptemos antes de su análisis.

\section{Notas}

(1) Este artículo es resultado del proyecto $\mathrm{N}^{\circ} 1120007$, “El procesamiento de enunciados condicionales en los estudiantes de Educación Media: un estudio a partir de la tarea de selección de Peter Wason", del Fondo Nacional de Desarrollo Científico y Tecnológico (FONDECYT), de la Comisión Nacional de Investigación Científica y Tecnológica (CONICYT), Ministerio de Educación, Gobierno de Chile. El autor, que es el investigador responsable del mencionado proyecto, agradece al programa FONDECYT y a las instituciones nombradas el financiamiento de este trabajo.

(2) Un trabajo emblemático en el que Goldberg y Freyd presentaron el Breve Estudio del Trauma con Traición es Goldberg y Freyd (2006). No obstante, como puede observarse, este trabajo es posterior al de DePrince (2005).

(3) Al fin y al cabo, DePrince notó que el trauma con traición y la disociación se hallaban vinculados.

(4) Ya a mediados de la década de los noventa, por ejemplo, Santamaría (1995) recogió las tesis fundamentales de la mayor parte de las teorías que, hasta ese momento, polemizaban acerca de la tarea de selección de las cuatro tarjetas de Peter Wason. En cierta medida, muchas de esas teorías continúan siendo apoyadas por sus seguidores en el presente. No nos hemos referido a ellas en este trabajo porque hacerlo excedería con mucho nuestros objetivos y podría hacer ingente nuestra labor. Sólo nos hemos centrado, 
como se ha indicado, en aquellos planteamientos que, desde nuestra óptica, tienen más directa relación con los temas fundamentales de estas páginas.

\section{Bibliografía}

Almor, A. y Sloman, S. A. 2000. Reasoning versus text processing in the Wason selection task -a non-deontic perspective on perspective effects. Memory and Cognition 28: 1060-1069.

Beller, S. y Spada, H. 2003. The logic of content effects in propositional reasoning: the case of conditional reasoning with a point of view. Thinking and Reasoning 9(4): 335-378.

Bernstein, E. M. y Putnam, F. W. 1986. Development, reliability, and validity of a dissociation scale. Journal of Nervous and Mental Disease 174: 727-735.

Briere, J. y Runtz, M. 1989. The Trauma Symptom Checklist (TSC-33): early data on a new scale. Journal of Interpersonal Violence 4: 151-163.

Cloitre, M; Scarvalone, P; Difede, J. 1997. Post-traumatic stress disorder, self and interpersonal dysfunction among sexually revictimized women. Journal of Traumatic Stress 10: 435-450.

DePrince, A. P. 2005. Social cognition and revictimization risk. Journal of Trauma and Dissociation 6(1): 125141.

Fiddick, L; Cosmides, L; Tooby, J. 2000. No interpretation without representation: the role of domain-specific representations and inferences in the Wason selection task. Cognition 77(1): 1-79.

Freyd, J. J. 1994. Betrayal-trauma: traumatic amnesia as an adaptive response to childhood abuse. Ethics and Behavior 4: 307-329.

Freyd, J. J. 1996. Betrayal trauma: the logic of forgetting childhood abuse. Cambridge: Harvard University Press.

Girotto, V. y Tentori, K. 2008. Is domain-general thinking a domain-specific adaptation? Mind and Society 7(2): 167-175.

Goldberg, L. R. y Freyd, J. J. 2006. Self-reports of potentially traumatic experiences in adult community sample: gender differences and test-retest stabilities of the items in a Brief Betrayal-Trauma Survey. Journal of Trauma and Dissociation 7(3): 39-63.

López, M. 2010. Los contratos sociales: ¿un nuevo formalismo ético? Alpha. Revista de Artes, Letras y Filosofía 30: 9-26.

López, M. 2011. ¿Existen reglas adaptativas en la mente humana? Una revisión crítica de las teorías cognitivas evolutivas por medio del enfoque de la perfección del condicional. Cinta moebio 41: 123-143.

Sandberg, D. A; Lynn, S. J; Matorin, A. I. 2001. Information processing of an acquaintance rape scenario among high -and low- dissociating college women. Journal of Traumatic Stress 14: 585-604. 
Santamaría, C. 1995. Introducción al razonamiento humano. Madrid: Alianza.

Sperber, D. y Girotto, V. 2002. Use or misuse of the selection task? Rejoinder to Fiddick, Cosmides, and Tooby. Cognition 85(3): 277-290.

Stone, V. E; Cosmides, L; Tooby, J; Kroll, N; Knight, R. 2002. Selective impairment of reasoning about social exchange in a patient with bilateral limbic system damage. Proceedings of the National Academy of Sciences 99: 11531-11536.

Wason, P. C. 1966. Reasoning. En: B. Foss. New horizons in psychology. Harmondsworth (Middlesex): Penguin, pp. 135-151.

Wason, P. C. 1968. Reasoning about a rule. Quarterly Journal of Experimental Psychology 20: 273-281.

Recibido el 17 de abril de 2012

Aceptado el 24 de julio de 2012 